\title{
Phone card application and authentication in wireless communications
}

\author{
C. H. Lee, M. S. Hwang and W. P. Yang \\ Department of Computer and Information Science \\ National Chiao Tung University, Hsinchu, Taiwan 30050, R.O.C. \\ e-mail: wpyang@cis.nctu.edu.tw \\ Fax: 886-35-721490 Tel: 886-35-712121 ext. 56617
}

\begin{abstract}
The Subscriber Identity Module (SIM) concept is widely used in many mobile communication systems. Some limitations and considerations of using SIM significantly reduce the availability and accessibility for the public in the wireless communications. The idea of using phone card for the public telephones is introduced for the mobile communications in this study. This new application is proposed to improve, in the meantime, to contain the merits of SIM. Major technical issues for using phone card includes the location tracking of the user, authentication of the phone card, and the billing scheme. In this study, three authentication approaches are also presented.
\end{abstract}

Authentication; Phone Card.

\section{Keywords}

\section{INTRODUCTION}

The Subscriber Identity Module (SIM) concept has been widely used in modern mobile communication systems. The SIM plays a major role in the security for both the subscribers and the networks (Mazziotto, 1992). The SIM has several major advantages in mobile communications (Mouly and Pautet, 1992). First, the SIM serves as a key to the communication equipment which charges to the account of SIM for its use. Once the SIM is removed, an equipment is no longer associated with the subscriber and the billing protection can be achieved. Second, the SIM is removable from one equipment to another and facilitates more flexible applications with the same billing account. Third, the SIM-roaming concept may provide the services at a much larger scale between systems based on different radio techniques. 
Nevertheless, the requirements of registration for the SIM and the ownership of a specified physical object (the subscriber's card) significantly reduce the availability and accessibility of SIM for the public in the wireless communications. The use of SIM is constrained by having a definite subscribing process and its economic concerns for short-term and more flexible uses of it. Those who use the wireless communications occasionally, wish to pay flexible and limited expenses at certain time and certain places, and hope to run less risk by losing the specific SIM card, will demand more flexibility for the application of mobile phones. As compared to a telephone system, the SIM card is more like a private phone and is not readily accessible to the public on the street. More versatile applications are deemed necessary and the idea of using phone card for the public telephones is thus introduced for the mobile communications in this study.

\section{PHONE CARD APPLICATIÓN}

The phone card, a new application of mobile phone, is proposed to improve and, in the meantime, to contain the merits of SIM. The phone card is a value-added alternative of SIM for the users. The scenario is that a user buys a phone card from a telecommunication company just as he owns a temporary SIM card and he can use any mobile phone at any place to make outgoing calls and to receive incoming calls with this card. The architecture of wireless mobile communication with phone card is shown in Figure 1.

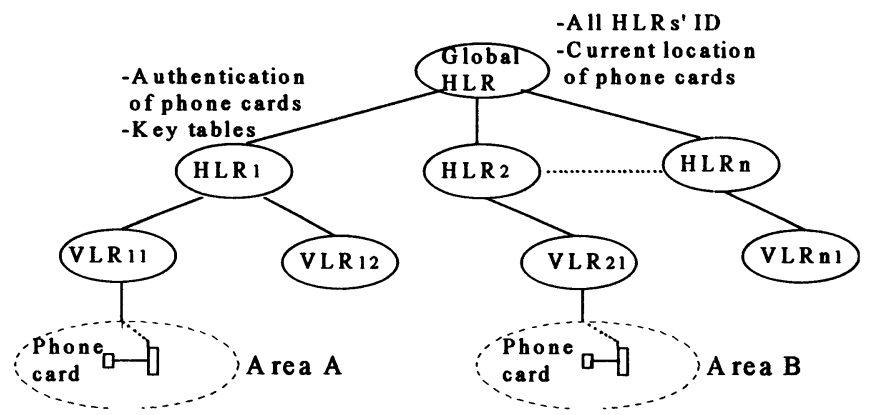

Figure 1 Architecture of wireless communication with phone card.

As illustrated in the Figure 1, the Global Home Location Register (Global HLR) holds all HLR's IDs and current locations of phone cards. The Global HLR plays an important role in location tracking when the user is roaming to different areas, i.e. the card-holder may roam from Area A to Area B. The HLR1 issues a phone card and stores $n$ keys in it. It keeps the key tables for its phone cards and has the responsibility to authenticate the phone cards. The key format of the phone card is drawn as

\begin{tabular}{|l|l|l|}
\hline HLR.id & Keys ..... & NB (or TU) \\
\hline
\end{tabular}


In this format, HLR.id is the global unique identifier who issues the cards. There are $\boldsymbol{n}$ keys generated by HLR and are stored in the card. NB stands for the Number of Bills, and TU is the Time Unit for a call. Both NB and TU can be used as the billing unit, where NB is used to charge by counts and TU is used to charge by time.

Major technical issues for using phone card includes the location tracking of the user, authentication of the phone card, and the billing scheme for Visited Location Register (VLR) (Wilkes, 1995). In the following, the descriptions of these issues are presented.

\section{LOCATION TRACKING OPERATION}

Location tracking is required when the network attempts to deliver a call to the card holder with a specific mobile phone. Figure 2 denotes the process of location tracking of the phone card. If a card-holder wants to receive an incoming call in a visited area, he may insert the card in any mobile phone and key-ins his Personal ID (PID) to register in this area. The visited HLR2 will pass his PID to the Global HLR to record the location of this specific user. Whenever and wherever an incoming call is for this PID, Global HLR first locates the user for the call, then the network can set up the trunk for the communication.

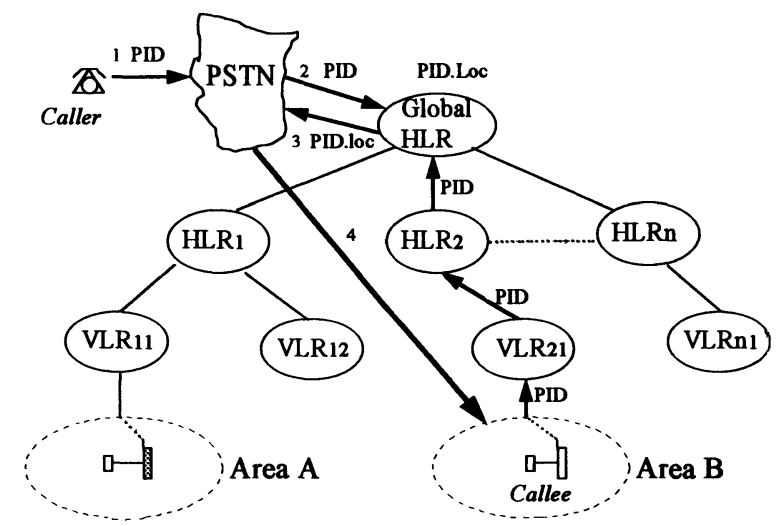

Figure 2 Process of location tracking of phone card.

\section{AUTHENTICATION OF PHONE CARD}

Authentication of the mobile user is a critical issue of correct billing for wireless communications (ETSI, 1993; Aziz and Diffie, 1994, Beheim, 1994; Beller, et al., 1993; Carlsen, 1994; Hagen, 1992; Lee et al., 1996; Molva et al., 1994). The process of authentication of card can protect both the card holders and the HLRs. The use of phone card in the holder's hometown is much less a problem than it is used out of town. If the holder uses the card in a visited area, visited HLR has to verify the card before a call can be delivered. 
Three authentication methods for the phone card are proposed in this article. The three approaches are the Key Table approach, the Signature approach and the Polynomial approach.

\subsection{Key table approach}

The first authentication approach is Key Table approach, as shown in Figure 3. HLR generates $n$ keys, stores them in the card, and maintains a table in itself. When card-holder wants to make a call at any place, i.e., his home domain or a visited domain, he inserts the card to a mobile phone and key-ins his PID, and then the information of authentication, i.e., (HLR.id, keyi, NB), can be routed to his home HLR. HLR checks if the keyin matches to one of the keys in the table.

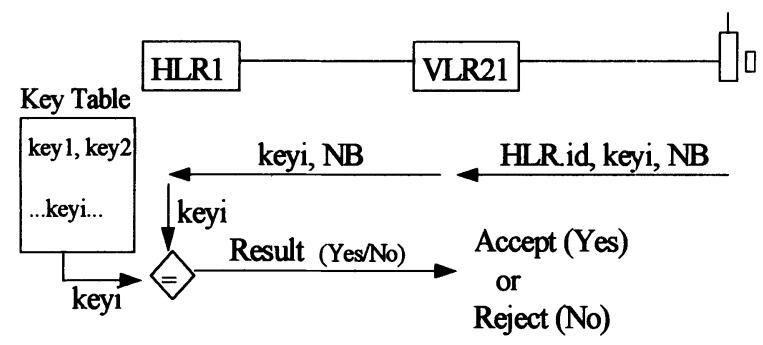

Figure 3 Key Table approach for authentication of phone card.

\subsection{Signature approach}

The second method is the Signature approach. Though the Key Table approach is simple, there is a problem for the plain key table in HLR to be exposed to and unlawfully used by unauthorized users. To avoid this concern, HLR will maintain a set of keywords instead of the plain keys in the table and stores the signatures of those keywords in the phone card. When a call is to be made, card sends the signature, i.e., Si, to HLR. HLR checks if there is any one of signatures of the keywords matches the Si . Figure 4 shows the Signature approach.

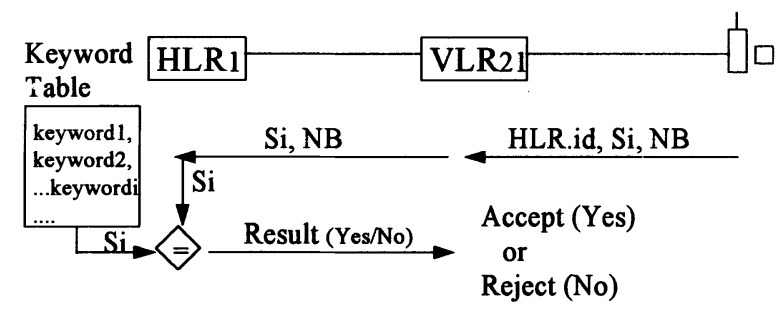

Figure 4 Signature approach for authentication of phone card. 


\subsection{Polynomial approach}

Both the Key Table and Signature approaches require storage space for the table. The third approach is proposed to use a polynomial instead of storing the tables in HLR. First, HLR selects a polynomial,

$h(x)=\left(x \oplus k_{1}\right)+x k 2 \operatorname{Mod} P=y$,

and its own secret keys, $k 1$ and $k 2$. HLR calculates $n$ pairs of $\left(x_{i}, y_{i}\right)$ from the polynomial and then stores those keys in the phone card. When the authentication takes place, HLR checks if the phone card holds the authorized key pair $\left(x_{i}, y_{i}\right)$. This approach is illustrated in Figure 5.

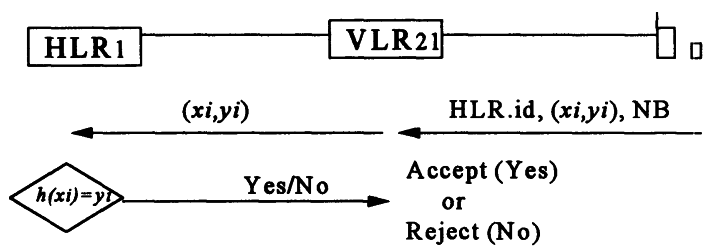

Figure 5 Polynomial approach for the authentication of phone card.

Comparisons are made for the three authentication methods. The Key Table Approach is simple but has to store the tables in HLR. Another drawback is that the card is much easier to be forged if the tables are obtained by unlawful users. The Signature Approach improves the first method by using signature to avoid the exposure of plain keys. However, it does not get rid of the need for the storage space. The Polynomial approach carries the advantages over storing key tables and leaking of sensitive information. One disadvantage in this approach is the problem of replaying. That is, how to avoid the key in phone card from using more than once? Our solution is that a bit map is set up in HLR to record the status of usage for those keys. Whenever a key is used for authentication, HLR sets the bit to 1 in the map. The HLR checks the correctness of the key and then checks if the key is used for more than once. The space for the bit map is considerably small when compared to the space for the Keyword Table in the Signature approach.

\section{BILLING SCHEME}

The third issue in the phone card application is the billing scheme. Billing scheme is designed to prevent the VLR of other domains from improper service charges. Two common occasions are often seen. One is that a VLR shares the key, which is transmitted from the card, with other VLRs. Another one is that VLR may overcharge for a given call. To avoid the keys being shared by several VLRs, we can check that none of the keys can be used more than once, i.e., to check the replaying. To prevent the overcharging problem, the authentication process must be done again when a TU is expired. 


\section{CONCLUSION}

Solutions for the location tracking, authentication, and the billing scheme make it more feasible for the application of phone card in mobile communications. The application of phone card is valuable for more diversified and extended uses of mobile phone with the merits but reduces the constraints of SIM card. Using a phone card is convenient for a person without carrying his mobile phone and the specific SIM card. Even if the phone card is lost, the financial risk would be reduced more to limited extents than that of losing a SIMs. The phone card application is expected to provide more channels for the public access and enhances the technological achievements of mobile telecommunications.

\section{REFERENCES}

European Telecommunications Standards Institute (1993) GSM 03.20: Security related network functions.

Aziz, A. and Diffie, W. (1994) Privacy and authentication for wireless local area network. IEEE Personal Communication, 1(1), 25-31.

Beheim, J. (1994) Security first in Europe's mobile communication. Telecom Report International, 17(1), 31-4.

Beller, M. J., Chang L. F. and Yacobi, Y. (1993) Privacy and authentication on a portable communications system. IEEE Journal on Selected Areas in Communication, 11(6), 821-9.

Carlsen, U. (1994) Optimal privacy and authentication on a portable communications system ACM Operation System Review, 16-23.

Hagen, R. (1992) Security requirements and their realization in mobile networks. XIV International Switching Symposium '92, 1, 127-131.

Lee, C. H., Hwang, M. S. and Yang, W. P. (1996) Authentication of mobile users in GSM system," Submitted for publication.

Mazziotto, G. (1992) The Subscriber Identity Module for the European digital cellular system GSM and other mobile communication systems. XIV International Switching Symposium, Yokohama, Japan.

Molva, R., Samfat D. and Tsudile, G. (1994) Authentication of mobile users. IEEE Network, March/April, 26-34.

Mouly M. and Pautet, M. B. (1992) The GSM System for Mobile Communications. ISBN: 29507190-0-7,.

Wilkes, J. E. (1995) Privacy and authentication needs of PCS. IEEE Personal Communication, 11-5.

\section{BIOGRAPHY}

Chrissy Chii-Hwa Lee received the BS from National Taiwan University, Taiwan, in 1976 and Master of Computer Science from Texas A\&M University, USA, in 1982. She is currently a $\mathrm{Ph} . \mathrm{D}$. candidate of Computer and Information Science in National Chiao Tung University, 
Taiwan. She joined to work on the projects of C3I System in Chung Shan Institute of Science and Technology (CSIST) under Department of Defense, Republic of China, since 1985. She was also the leader of Management Information Systems of CSIST since 1988. Her research interests include database security, mobile computing, mobile communications and database systems.

Min-Shiang Hwang received the BS in EE from National Taipei Institute of Technology, Taiwan, in 1980; the MS in EE from National Tsing Hua University, Taiwan, in 1988; and the Ph.D. in Computer and Information Science from National Chiao Tung University, Taiwan, in 1995. He was the leader of the Computer Center at Telecommunication Laboratories (TL), Ministry of Transportation and Communications. He was also a project leader for research in computer security at TL since 1990 . He is a member of IEEE and ACM. His research interests include cryptography, data security, database systems, and network management.

Wei-Pang Yang received the BS in mathematics from National Taiwan Normal University in 1974, and the MS and Ph.D. from the National Chiao Tung University (NCTU) in 1979 and 1984 , respectively, both in computer engineering. He has been on the faculty of the Department of Computer Science and Information Engineering at NCTU, Taiwan, since 1979. In 1988, he joined the Department of Computer and Information Science at NCTU. His research interests include database theory, database security, object-oriented database, image database, and Chinese database retrieval systems. Professor Yang is a senior member of IEEE and a member of ACM. 